\title{
Design and optimization of a wideband metasurface for utilization in a highly efficient terahertz reflectarray antenna
}

\section{Raziyeh Sharifi}

Shiraz University of Technology

Raheleh Basiri ( $\square$ r.basiri@sutech.ac.ir)

Shiraz University of Technology https://orcid.org/0000-0002-9111-8375

Ehsan Zareian-Jahromi

Shiraz University of Technology

\section{Research Article}

Keywords: Efficiency, Metasurface, Optimization, Reflectarray, Terahertz

Posted Date: June 10th, 2021

DOl: https://doi.org/10.21203/rs.3.rs-358671/v1

License: (c) (i) This work is licensed under a Creative Commons Attribution 4.0 International License.

Read Full License 
Name of Authors:

1- Raziyeh Sharifi

2- Raheleh Basiri (Corresponding author)

3- Ehsan Zareian-Jahromi

Title:

Design and optimization of a wideband metasurface for utilization in a highly efficient terahertz reflectarray antenna

Affiliation:

Department of Electrical Engineering, Shiraz University of Technology, Shiraz 7155713876, Iran

Email address:

1- Raziyeh Sharifi: R.Sharifi@sutech.ac.ir

2- Raheleh Basiri (Corresponding author): r.basiri@sutech.ac.ir

3- Ehsan Zareian-Jahromi: zareian@sutech.ac.ir

\section{6-digit ORCID}

1- Raheleh Basiri (Corresponding author): 0000-0002-9111-8375

2- Ehsan Zareian-Jahromi: 0000-0001-5885-8665 


\title{
Design and optimization of a wideband metasurface for utilization in a highly efficient terahertz reflectarray antenna
}

\author{
Raziyeh Sharifi, Raheleh Basiri* and Ehsan Zareian-Jahromi \\ Department of Electrical Engineering, Shiraz University of Technology, Shiraz 7155713876, Iran \\ *Corresponding author: $\underline{\text { r.basiri@sutech.ac.ir }}$
}

\begin{abstract}
In this manuscript, two wideband single layer reflectarray antennas are designed utilizing an optimization-based metasurface in terahertz $(\mathrm{THz})$ regime. It is demanded to design a metasurface with wide phase variation range in a broad frequency region of terahertz band. The proposed metasurface is designed based on Random Hill Climbing optimization algorithm whereas a multiobjective fitness function is defined to consider the desired characteristics. A provided link between Matlab and HFSS softwares is utilized to define and simulate various metasurfaces. The finalized cell has considerable wide phase variation $\left(\geq 600^{\circ}\right)$ and notable bandwidth of $30.76 \%(1.1-1.5 \mathrm{THz})$ whereas the mean value of magnitude variation of reflection coefficient is $-0.42 \mathrm{~dB}$. Two square and circular metasurfaces are designed based on the optimized cell and illuminated using a THz feeding horn antenna. The angles of incident and reflected waves are considered equal to zero. The simulation results confirm 3-dB gain bandwidths of $20.3 \%$ and $20.4 \%$ for square and circular reflectors, respectively. Moreover, the considerable efficiencies of $45.67 \%$ and $46.27 \%$ are achieved for square and circular arrays, consequently. The maximum gain of square array with 361 elements is $25.9 \mathrm{~dB}$ whereas it is equal to $24.9 \mathrm{~dB}$ for circular metasurface including 277 unit cells.
\end{abstract}

Keywords: Efficiency, Metasurface, Optimization, Reflectarray, Terahertz.

\section{1-Introduction}

Terahertz (THz) band is located at the end of microwave band before the start of infrared band in the electromagnetic spectrum and hence, benefits from the advantages of these two bands. THz spectrum covers the frequency range from $0.1 \mathrm{THz}$ to $10 \mathrm{THz}$ [1]. Considering practical perspective among various frequency ranges, $\mathrm{THz}$ antennas have attracted much interest in the last decade [2, 3].

One of the solutions for increasing data rate in new generation of telecommunications is the use of higher frequencies, especially the terahertz band. Moreover, utilizing $\mathrm{THz}$ band has additional applications including multimedia broadcasting [4,5], wireless technology [2,6], and medical imaging [7, 8]. One of the main challenges of utilizing $\mathrm{THz}$ regime for communications is high attenuation rate of $\mathrm{THz}$ waves in the atmosphere. To overcome this, it is necessary to design high gain antennas [9-11].

Many types of high gain antennas including phased array [12], parabolic [13], and lens antennas [14-18] are applicable in THz communications. Typically, these antennas are highly efficient and fall into the category of high-gain antennas. Each type of these antennas has individual disadvantages and advantages. The parabolic reflectors have high manufacturing complexity due to their special geometry according to curvature of the metal plate, high weight and volume. The phase array antennas are not suitable for common telecommunications due to the complexity of demanded power network, construction cost, as well as excessive losses in transmitter and 
receiver parts. The drawbacks of these two types of antennas have considerably resolved in reflectarray antennas, which are combination of classic reflector antennas and phase arrays [19-22].

The disadvantages of the reflector and phase array antennas have been a challenge until 1963 where an article entitled as "The reflectarray antenna" was published by Berry [23]. The advantages of parabolic reflector and phased array antennas are combined in the proposed antenna, whereas the problems with these two antennas are eliminated in the reflectarray antenna. In 1978, the reflectarray antenna is developed based on utilizing microstrip patch elements [24]. In addition to its simple planar construction, the antenna has remarkable benefits including low loss, high gain, low-profile, low weight, easy design and fabrication, together with potential for reconfigurability and dynamic control capability $[19,20]$.

Reflectarray antennas include a planar array of radiating elements excited by a feed antenna. The shape and size of these elements are designed and modified to create different reflection phases. Therefore, the reflected waves from the reflector array can be collected in a desired direction resulting in a single main lobe with a specific beamwidth. In fact, the variation of dimensions and shape of cells is used to compensate different phase values caused by variant encountered distances from the feed antenna [25, 26].

The performance of a reflector antenna is strongly dependent on characteristics of including cells. The cell shape is the most effective parameter determining the cell property. Therefore, the cell design is a critical stage in the evaluation of a reflectarray antenna. Some optimization algorithm may be utilized to develop an optimized cell with specific characteristics. Random Hill Climbing (RHC) optimization algorithm is one of the most efficient utilized algorithms [27].

The RHC is a powerful optimization algorithm that is moving towards increasing the value of an objective function. This algorithm has a repetitive process starting with a desired or random solution and then, provides a better response by changing the position of the bits in a binary string. This method continues until further progress is possible [28].

In this paper, a broadband single layer unit cell with wide phase variation is designed based on RHC optimization algorithm. The proposed cell is utilized in two wideband terahertz reflectarray antennas. The paper is redacted as following. Some design aspects are explained in Section 2. The optimization algorithm and corresponding fitness function are comprehensively investigated in Section 3. The simulation results of proposed unit cell are studied in Section 4 whereas two reflectarray antennas with square and circular arrangements of the proposed unit cell are designed and presented in Section 5. Finally, the manuscript is concluded in Section 6.

\section{2- Design Aspects}

One of the main drawbacks of the reflectarray antenna design is its narrow bandwidth. This is due to narrow band feature of involved unit cells. Therefore, this problem can be removed by utilizing a wideband unit cell [29]. Moreover, it is desired to have a high gain reflectarray antenna in many applications [10]. This goal can be achieved utilizing large reflector planes with numerous cells. The range of phase variation of a cell is usually about $360^{\circ}$ which is not sufficient for large reflectarray antennas. Low phase variation of utilized unit cells in large reflectarray antennas results in a sudden dimension variation at the corner elements. Therefore, the assumed periodic boundary conditions at simulation of utilized cell would be no longer satisfied and hence, the cell performance in the metasurface would be very different from that in a periodic structure. 
In addition to unit cell bandwidth, the phase delay caused by the physical distances between the feed antenna and the corners of the reflector plane is another important parameter reducing the bandwidth of a reflectarray antenna. This delay causes to reduce gain of reflectarray antenna. Moreover, the radiation pattern of reflectarray antenna degrades over a wide frequency range. The angles of incident and reflected waves are also effective in determining the phase of each cell. In other words, a phase variation range of $360^{\circ}$ may be sufficient for some angles of incidence and reflection whereas it is not adequate for another ones.

Therefore, the cells with phase variation range of greater than $360^{\circ}$ should be designed to solve the problem of narrow bandwidth encountered in large reflectarray antennas. A noteworthy point in the design of cells with a wide range of phase variation is relatively gentle slope of the phase-scale diagram.

In this paper, a broadband single-layer cell with a high phase variation is designed utilizing RHC optimization algorithm. Then, the phase response analysis and other features of the single cell are presented. Finally, two reflectarray antennas with good performances and high efficiency values are designed based on the optimized cell. The feed antenna is a horn antenna whereas the corresponding design procedure is described in the following section.

The demanded range of $1 \mathrm{THz}$ to $1.5 \mathrm{THz}$ is considered in the design of unit cell and reflectarray antenna. A wideband unit cell with high phase variation range is achieved by definition of a multi-objective fitness function.

\section{3- RHC optimization algorithm}

As it is previously mentioned, the RHC optimization algorithm is utilized to define various unit cells. Different stages of definition, analysis and simulation of an arbitrary cell are expressed in the following subsections.

\section{3-1 Unit cell definition}

As shown in Fig. 1, a grid including $16 \times 16$ small squares is used to define a single-layer cell. Each small square, which will be called as a pixel, is $6.25 \times 6.25 \mu \mathrm{m}^{2}$.

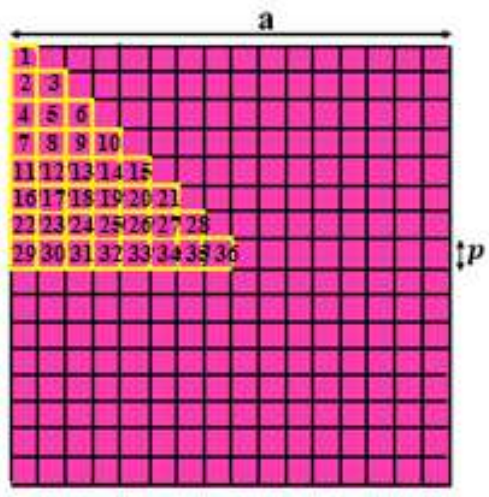

Fig. 1: Definition of the unit cell using a $16 \times 16$ square network

The cell is divided into 256 pixels. Due to symmetry of the structure, one-eighth of the cell is defined by a 36-bit string and hence, the rest is completed by repeating this part.

\section{3-2 Determination of the fitness function}

In this sub-section, the fitness function consisting of several parts is defined to consider the desired goals. In the first part, it is desired to achieve a linear phase-scale diagram for the frequencies between $1 \mathrm{THz}$ to $1.5 \mathrm{THz}$ 
whereas the range of phase variation should be more than $600^{\circ}$. This condition is expressed mathematically in equation (1):

$$
\begin{aligned}
\mathrm{D}_{1} & =\frac{600^{\circ}}{\text { scale }} \\
\mathrm{Q}_{\mathrm{k}} & =\left(\text { Average }\left(\mathrm{R}_{\mathrm{k}}\right)-\mathrm{D}_{1}\right) \\
\mathrm{Q}_{\mathrm{k}} & \leq 0
\end{aligned}
$$

In the equation (1), $\mathrm{k}$ is the $\mathrm{k}$-th desired frequency between $1 \mathrm{THz}$ and $1.5 \mathrm{THz}$ whereas the frequency step is considered to $0.1 \mathrm{THz}$. The slope of the ideal phase-scale diagram is designated to $-600^{\circ} /$ scale (Fig. 2). $\mathrm{Q}_{\mathrm{k}}$ is the difference between the mean slope of phase-scale diagram of $k$-th desired frequency, $\mathrm{R}_{\mathrm{k}}$, and the slope of ideal curve in Fig. 2.

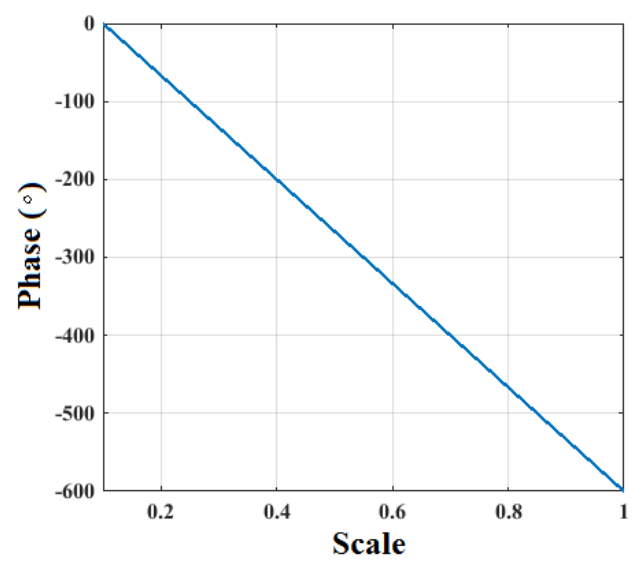

Fig. 2: The ideal phase-scale diagram of the unit cell

The second part of the objective function is stated in equation (2). It is demanded that the average amplitude of the reflection coefficient considering different scales at all desired frequencies should be greater than $-1 \mathrm{~dB}$.

$$
\begin{aligned}
& \mathrm{X}_{1}=\text { Average }\left(\operatorname{mag}\left(\mathrm{S}_{11}\right)\right) \\
& \mathrm{X}_{1} \geq-1 \mathrm{~dB}
\end{aligned}
$$

Other restrictions are also considered in addition to the above-mentioned conditions. The minimum relative bandwidth of the cell should not be less than $25 \%$. Therefore, the acceptable cases are limited to the following four states:

$$
\begin{aligned}
& \mathrm{Zl}_{\mathrm{k}, \mathrm{m}}=\left|\mathrm{AR}_{\mathrm{k}}-\mathrm{AR}_{\mathrm{k}-\mathrm{m}}\right| \mathrm{k}=2,3, \ldots, 6 ; \mathrm{m}<\mathrm{k} \\
& \mathrm{Zl}_{\mathrm{k}, \mathrm{m}} \leq 150 \\
& \mathrm{Z}_{\mathrm{k}, \mathrm{m}}=\left|\mathrm{AR}_{\mathrm{k}}-\mathrm{AR}_{\mathrm{k}-\mathrm{m}}\right| \mathrm{k}=2,3, \ldots, 5 ; \mathrm{m}<\mathrm{k} \\
& \mathrm{Z}_{\mathrm{k}, \mathrm{m}} \leq 150 \\
& \mathrm{Z}_{\mathrm{k}, \mathrm{m}}=\left|\mathrm{AR}_{\mathrm{k}}-\mathrm{AR}_{\mathrm{k}-\mathrm{m}}\right| \mathrm{k}=3,4, \ldots, 6 ; \mathrm{m}<\mathrm{k} \\
& \mathrm{Z}_{\mathrm{k}, \mathrm{m}} \leq 150 \\
& \mathrm{Z}_{\mathrm{k}, \mathrm{m}}=\left|\mathrm{AR}_{\mathrm{k}}-\mathrm{AR}_{\mathrm{k}-\mathrm{m}}\right| \mathrm{k}=2, \ldots, 4 ; \mathrm{m}<\mathrm{k} \\
& \mathrm{Z}_{\mathrm{k}, \mathrm{m}} \leq 150
\end{aligned}
$$


In all relations of 3 to $6, \mathrm{AR}_{\mathrm{k}}$ is the average slope of the phase-scale diagram for $\mathrm{k}$-th desired frequency.

The absolute value $\mathrm{Zi}_{\mathrm{k}, \mathrm{m}}$ is the difference between the mean values of the slopes for different frequencies. In conditions 3 to 6 , the value of m must be less than $\mathrm{k}$ where it is between 1 and 6 (Table 1 ).

Table 1: The relation between parameter $k$ and desired frequencies between $1 \mathrm{THz}$ to $1.5 \mathrm{THz}$

\begin{tabular}{c|c|c|c|c|c|c}
$\mathbf{k}$ & 1 & 2 & 3 & 4 & 5 & 6 \\
\hline frequency (THz) & 1 & 1.1 & 1.2 & 1.3 & 1.4 & 1.5
\end{tabular}

The conditions (3) to (6) indicate that in which frequency region the difference between the average of corresponding phase-scale variation diagrams in adjacent frequencies is less than or equal to 150 degrees. This condition causes a relative similarity of the phase change curves with each other. In all four cases, the relative bandwidth of the cell is higher than $25 \%$.

The implementation of the conditions (3)-(6) is explained in the following. At first, the equation (3) is examined. If this condition is met, the produced structure has acceptable performance at frequencies between $1 \mathrm{THz}$ and 1.5 $\mathrm{THz}$ else equation (4) will be considered. If the condition (4) is satisfied, the structure has acceptable characteristic in the frequency range between $1 \mathrm{THz}$ and $1.4 \mathrm{THz}$. In the case that none of equations (3) and (4) are met, equation (5) has been examined and this process continues similar to the previous two conditions until the appropriate answer is obtained for the fitness function.

\section{3-3 Optimized unit cell}

Based on fitness function definition, the optimal cell obtained after 4000 steps of program execution is defined by the resulting 36-bit code string of [001010010101010000000010011101010101]. The schematic view of obtained cell is presented in Fig. 3 .

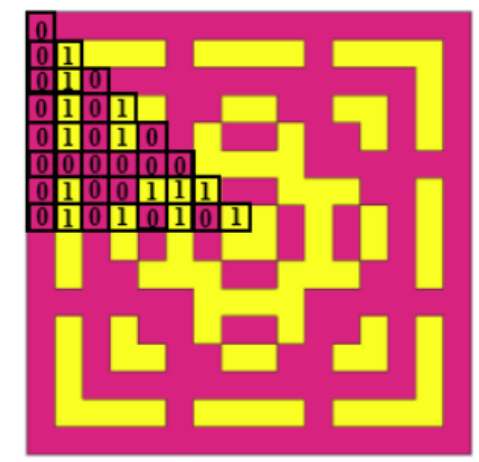

Fig. 3: Optimized unit cell obtained from RHC optimization process

The substrate of the cell is made of Silicon with dielectric constant of 11.9 and loss tangent of 0.001. Moreover, the gold layer with surface impedance of $0.287+\mathrm{j} 0.335 \Omega$ is utilized as metallic parts of the cell.

\section{4- Simulation results of the optimized cell}

Fig. 4 shows the phase variation diagram of optimized cell in terms of various scales of the metallic section of the cell considering desired frequencies between $1.1 \mathrm{THz}$ and $1.5 \mathrm{THz}$. The optimized structure meets condition (5) whereas the corresponding bandwidth is $30.76 \%$. Moreover, the maximum phase variation is $738^{\circ}$ at $1.5 \mathrm{THz}$ and more than $600^{\circ}$ at other frequencies. 


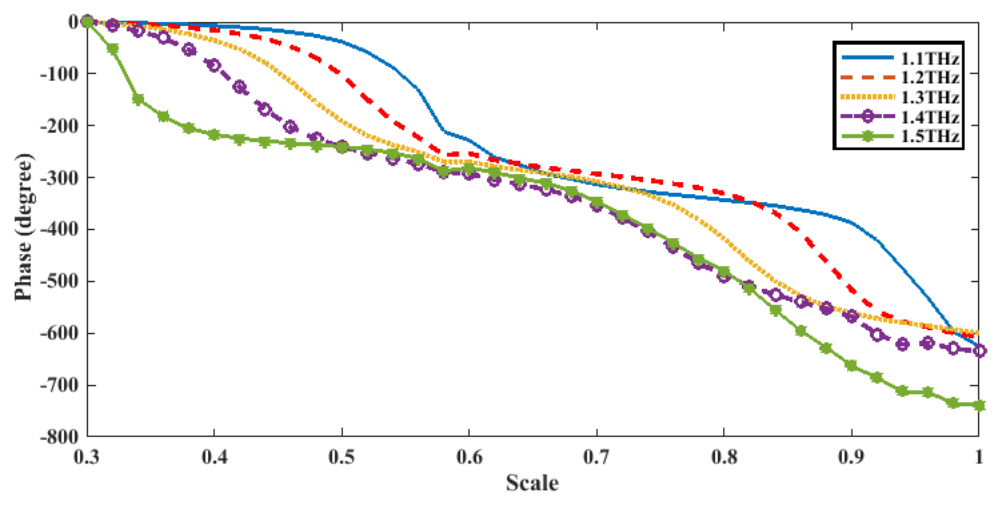

Fig. 4: Phase-scale diagrams of the optimized cell considering different frequencies between 1.1-1.5 THz

The mean values of corresponding reflection coefficient considering various scales at different desired frequencies are tabulated in Table 2.

Table 2: Reflection coefficient mean values of achieved cell in Fig. 3 at different frequencies

\begin{tabular}{c|c|c|c|c|c} 
Frequency $(\mathbf{T H z})$ & 1.1 & 1.2 & 1.3 & 1.4 & 1.5 \\
\hline Mean $\mathrm{S}_{11}(\mathbf{d B})$ & -0.39 & -0.42 & -0.36 & -0.38 & -0.36
\end{tabular}

To provide a conceptual explanation for the optimized cell characteristics, the cell evolution is examined in three steps according to Fig. 5. Afterwards, corresponding phase-scale diagrams are depicted in Fig. 6.

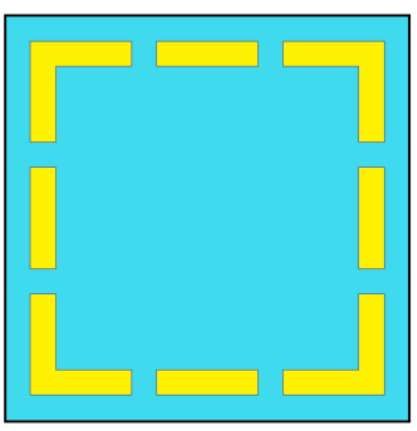

(a)

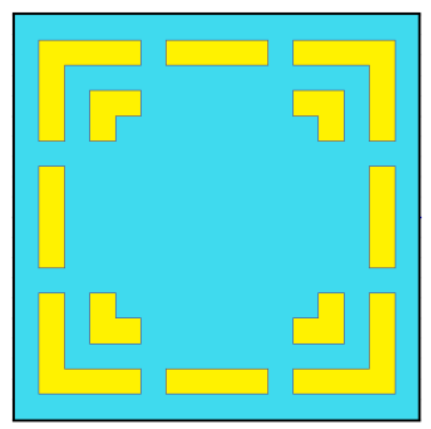

(b)

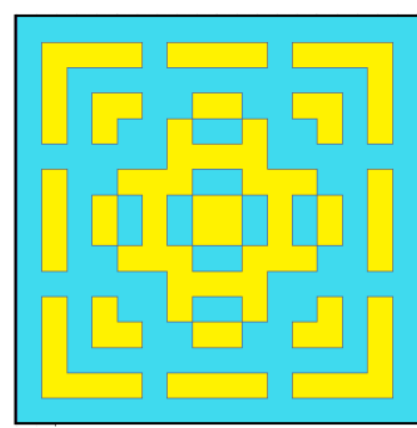

(c)

Fig. 5: Evolution of the optimized cell in different stages

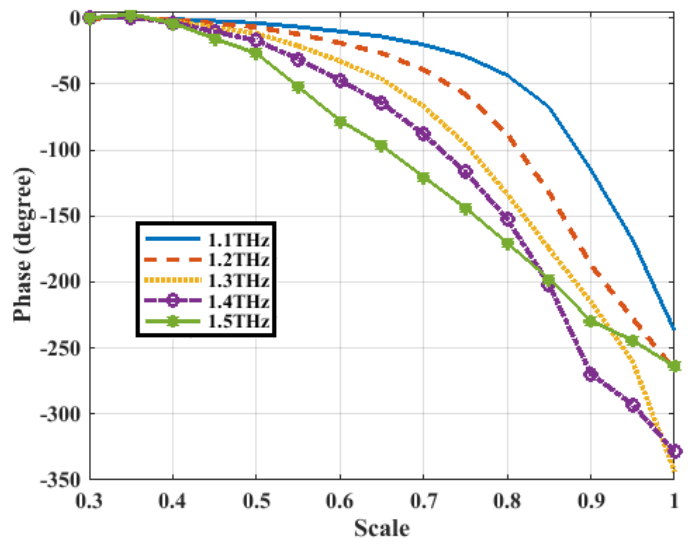

(a)

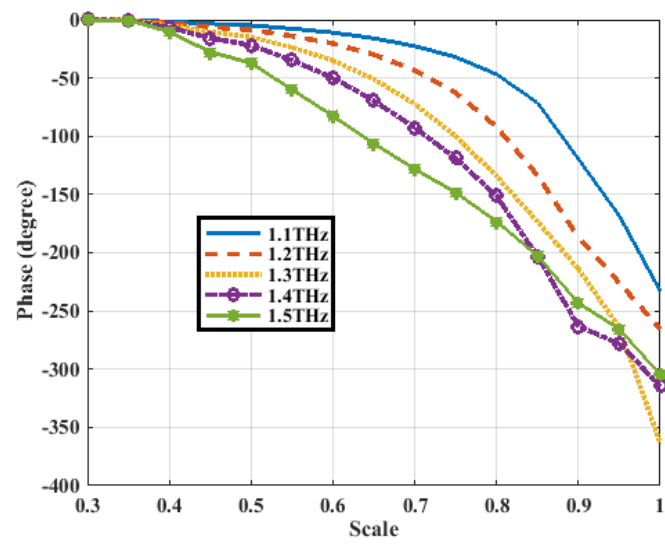

(b) 


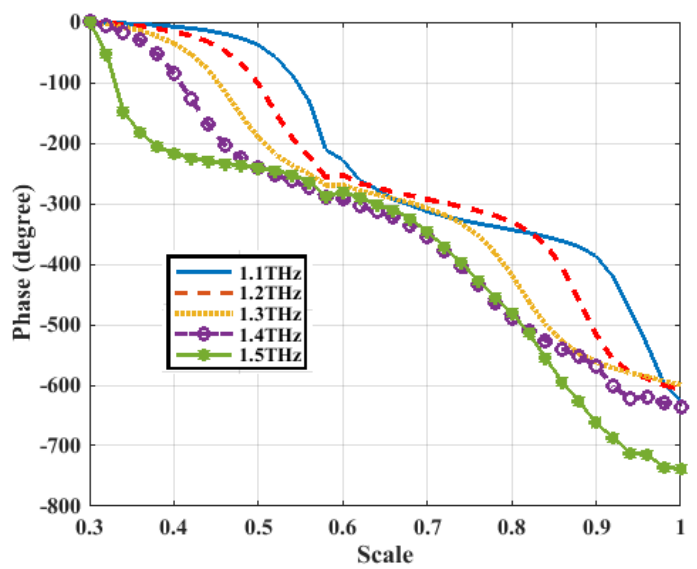

(c)

Fig. 6: Corresponding phase-scale diagrams of the cell in different stages of Fig. 5

According to Fig. 6, the phase variation range increases in Fig. 6c with respect to Fig. 6a. Moreover, the phasescale characteristics become closer at adjacent frequencies in the last state of cell evolution. Therefore, an increased bandwidth is obtained utilizing the structure in Fig. 5c. Surface current distributions on the metallic parts of the optimized cell are investigated considering different frequencies (Fig. 7).

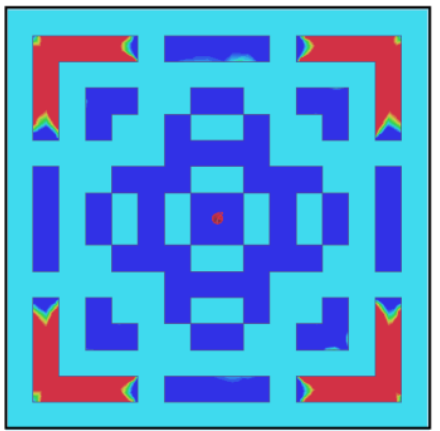

(a)

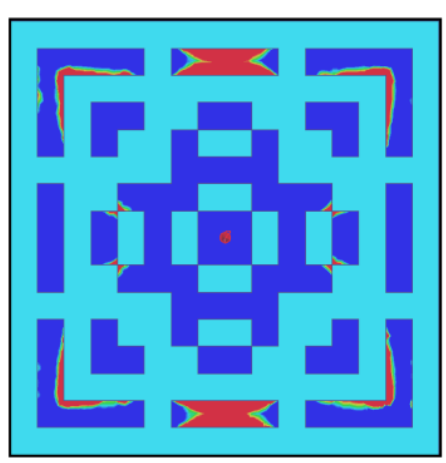

(d)

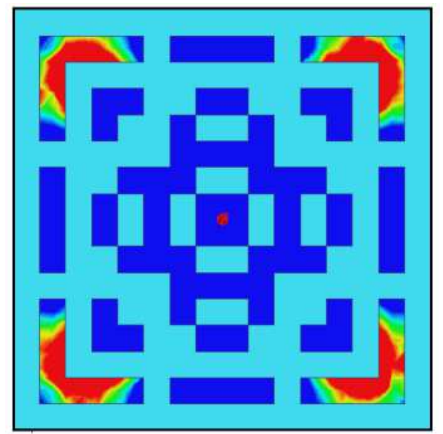

(b)

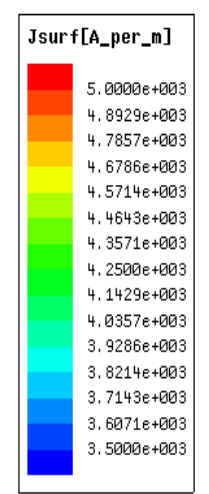

.

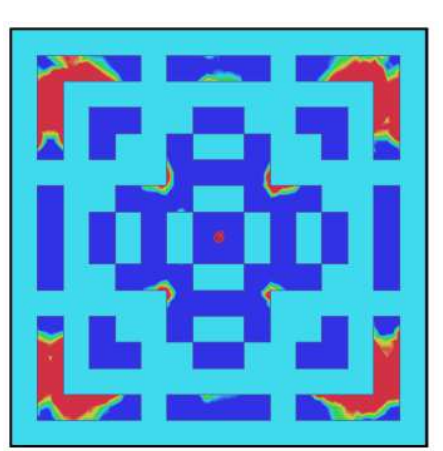

(c)

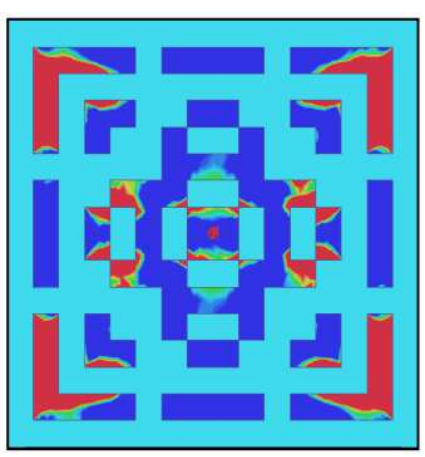

(e)

Fig. 7: Current distributions of the structure in Fig. 5c at: (a) $1.1 \mathrm{THz}$, (b) $1.2 \mathrm{THz}$, (c) $1.3 \mathrm{THz}$, (d) $1.4 \mathrm{THz}$, (e) $1.5 \mathrm{THz}$

It is evident that cell corners are more excited at lower frequencies whereas the central parts are also excited at higher frequencies. 


\section{5- Design of reflectarray antenna}

In this section, two different metasurfaces are designed based on the proposed optimized cell. In the first design, a square array design is considered whereas corresponding cells are arranged on a circular reflector plane in second design. The designed metasurfaces are utilized in two reflectarray antennas which are illuminated by a horn antenna located at a suitable distance with respect to the reflecting plane.

The incident angle is considered equal to zero. Moreover, the radiation characteristics of illuminating horn antenna are studied in the following section.

\subsection{Specifications of feed horn antenna}

In this sub-section, a broadband horn antenna is designed to excite two proposed reflectarray antennas. The schematic view of proposed horn antenna is depicted in Fig. 8.

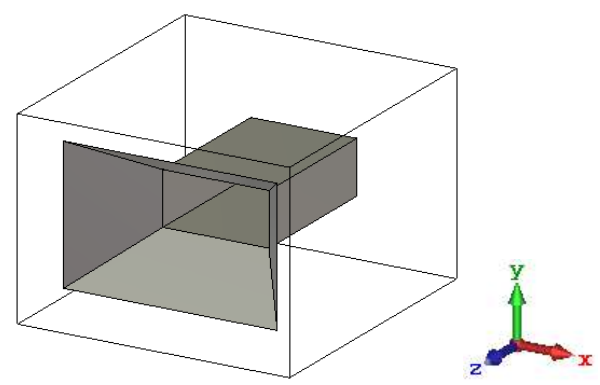

Fig. 8: Scheme of the designed feed horn antenna

The horn antenna aperture size is equal to $423 \times 268 \mu \mathrm{m}^{2}$ whereas the operation frequency range covers the demanded frequency band according to proposed reflectarray antennas. The radiation pattern of the proposed feed antenna at $1.3 \mathrm{THz}$ is shown in Fig. 9.

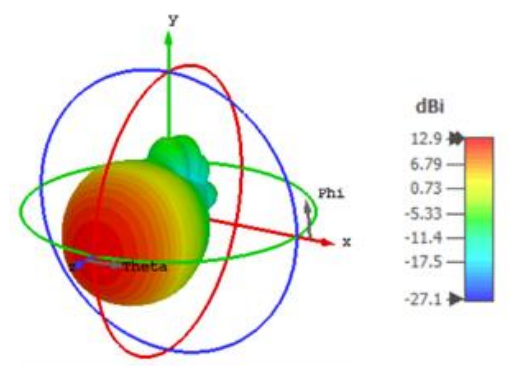

Fig. 9: Radiation pattern of the proposed horn antenna in Fig. 8 at $1.3 \mathrm{THz}$

The maximum gain of the designed feed antenna is equal to $12.9 \mathrm{~dB}$ at $1.3 \mathrm{THz}$. The corresponding gain and return loss variations are illustrated in Fig. 10 for frequencies between $0.9 \mathrm{THz}$ to $1.5 \mathrm{THz}$. 


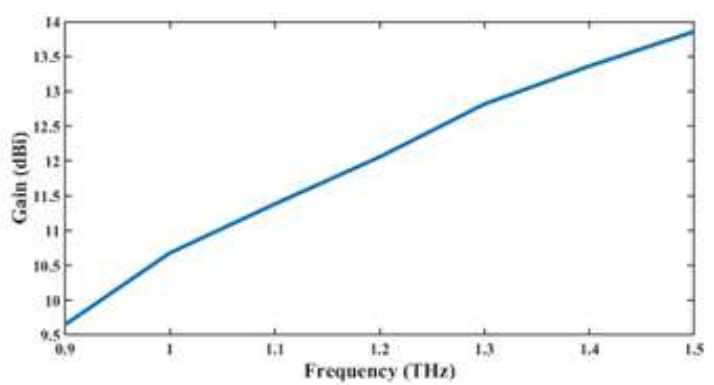

(a)

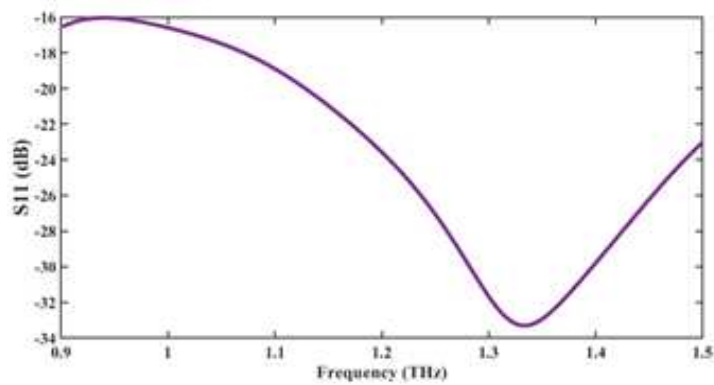

(b)

Fig. 10: Specifications of the feed horn antenna, (a) gain and (b) return loss variations versus frequency

The proposed horn antenna is utilized to feed two designed reflectarray antennas. These reflectarray structures are comprehensively investigated in the following sub-sections.

\section{5-2 First reflectarray structure: square layout}

In the first design, 361 optimized cells are arranged in a $19 \times 19$ square metasurface. The angles of incident and reflected waves are assumed to be zero whereas the reflector plane is $1900 \times 1900 \mu \mathrm{m}^{2}$. The feed antenna is positioned at the optimized distance of $1900 \mu \mathrm{m}$ with respect to the center of the reflector plane, which is equal to the focal point of the array at the central frequency of $1.3 \mathrm{THz}$.

\section{5-2-1 Cell arrangement on the square array}

The required phase of each unit cell located at a specific position on the metasurface is calculated as follows [30],

$$
\varphi_{\mathrm{RA}}=\varphi_{\mathrm{pp}}+\varphi_{\mathrm{spd}}=\mathrm{k}_{0}\left(\mathrm{R}_{\mathrm{i}}-\sin \theta_{0}\left(\mathrm{x}_{\mathrm{i}} \cos \varphi_{0}+\mathrm{y}_{\mathrm{i}} \sin \varphi_{0}\right)\right)
$$

The reflecting phase of each cell is denoted by $\varphi_{\text {spd }}$ compensating the phase delay due to the distance of the feed antenna to the corresponding element of the array. Moreover, to focus the reflected beam into a specific direction of $\left(\theta_{0}, \varphi_{0}\right)$, a progressive phase must be added to the aperture that is shown by $\varphi_{\mathrm{pp}}$ in equation (7). Therefore, the required phase of each element in the aperture, $\varphi_{\mathrm{RA}}$, is a combination of two compensating and progressive phases.

In equation (7), $\mathrm{R}_{\mathrm{i}}$ is the distance from the center of feed antenna to the ith element and $\mathrm{k}_{0}$ is the free space wave number at the central frequency. Moreover, the position of each element is expressed by $\left(\mathrm{x}_{\mathrm{i}}, \mathrm{y}_{\mathrm{i}}\right)$. The phase distribution diagram on the surface of the $19 \times 19$ reflectarray antenna at the central frequency of $1.3 \mathrm{THz}$ is presented in Fig. 11. 


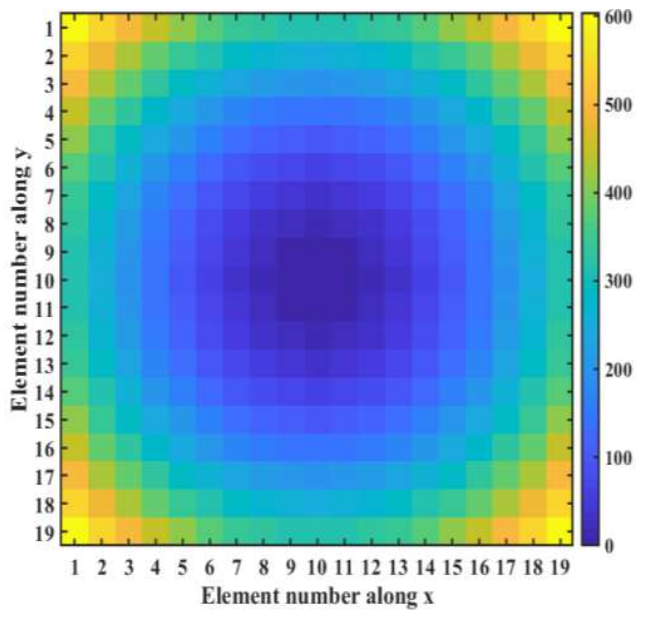

Fig. 11: Phase distribution diagram of the cells located on the metasurface at the central frequency of $1.3 \mathrm{THz}$ The phase distribution on the metasurface includes one-eighth symmetry. According to Fig. 11, the required phase values in Fig. 11, are provided by scaling the metallic pattern of designed cell. The phase-scale diagram according to Fig. 4 is utilized to determine the required scales for demanded phase values in Fig. 11. The corresponding scales of each cell are presented in Table 3 . The phase origin is the cell located at the center of the reflectarray, whereas the phase shifts of other cells are considered with respect to this central cell. Due to symmetry of the structure, it is sufficient to provide the dimensions of 55 cells located in one eighth of the array (Table 3).

Table 3: Corresponding scales of each cell in one eighth of the $19 \times 19$ reflectarray antenna

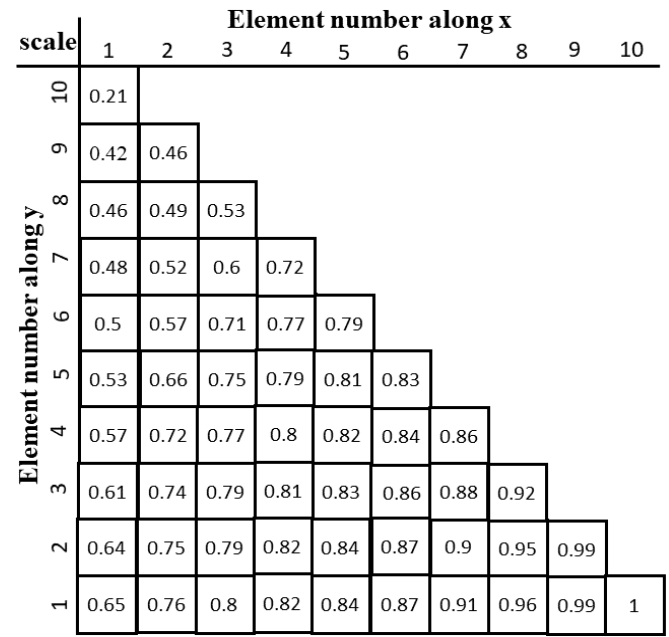

The scheme of finalized reflectarray antenna consisting of 361 optimized cells is demonstrated in Fig. 12. 


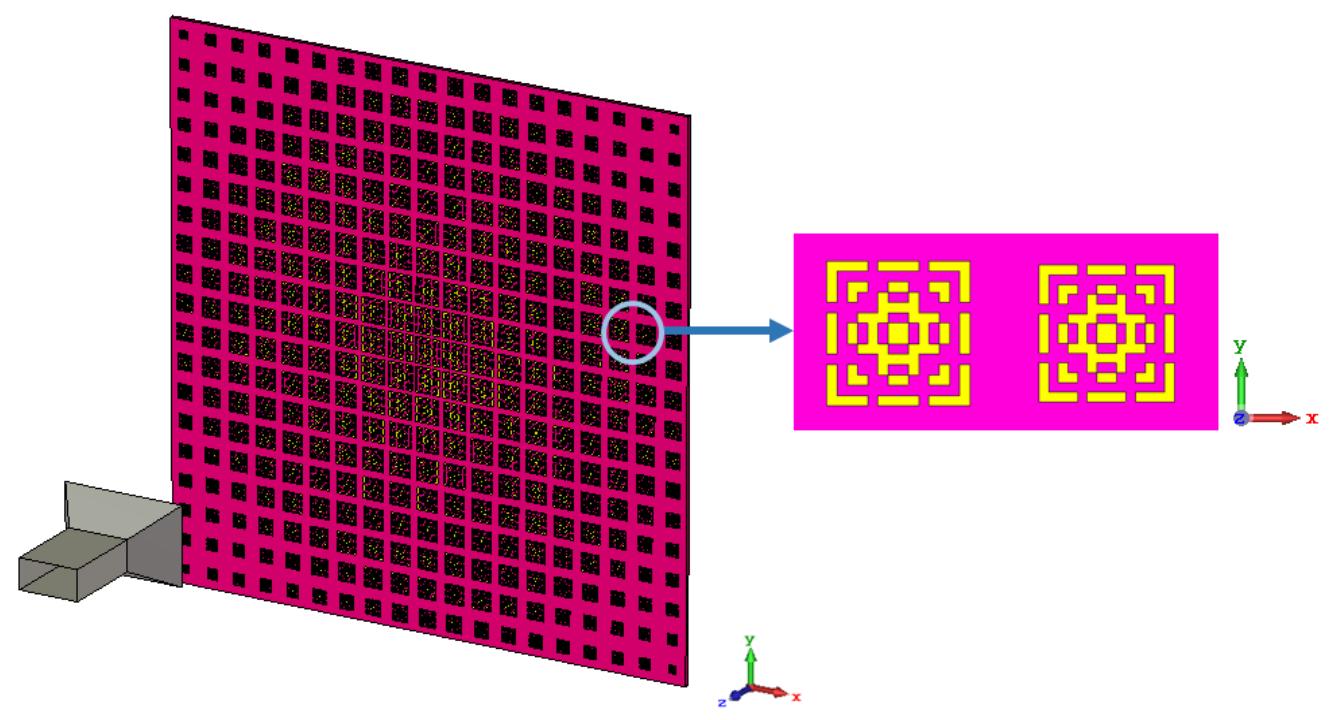

Fig. 12: Total view of the $19 \times 19$ designed square reflectarray antenna with feed antenna

\section{5-2-2 Simulation and performance analysis}

The maximum gain of the $19 \times 19$ square reflectarray antenna is $25.9 \mathrm{~dB}$ at the central frequency of $1.3 \mathrm{THz}$. The gain variation of designed reflectarray antenna is compared to the feed antenna in Fig. 13. The $3 \mathrm{~dB}$ gain bandwidth of designed reflectarray antenna is $20.3 \%(1.15-1.41 \mathrm{THz})$. The maximum efficiency of the proposed reflectarray antenna at $1.3 \mathrm{THz}$ is calculated by equation (8) resulting to a $45.67 \%$ efficiency value [30]:

$$
\eta_{\text {aperture }}=\mathrm{G} \frac{\lambda^{2}}{4 \pi \mathrm{A}}
$$

where $\lambda$ is corresponding wavelength at $1.3 \mathrm{THz}$ and $\eta_{\text {aperture }}$ is corresponding antenna efficiency.

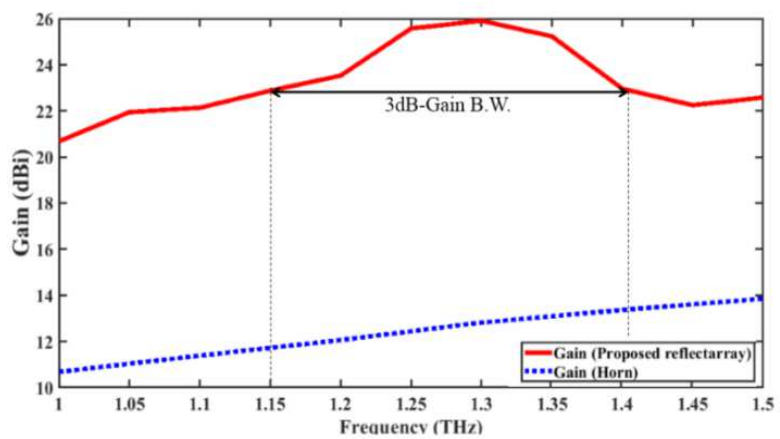

Fig. 13: Comparison between gain of designed reflectarray antenna and that of feed horn antenna at the desired

$$
\text { frequency band }
$$

Due to wide provided phase variation range of the optimized cell $\left(\geq 600^{\circ}\right)$, no suddenly change would be occurred in the size of the adjacent cells. Therefore, the bandwidth of the reflectarray is desirable with respect to similar structures, e.g. [10]. The simulated radiation patterns of the antenna at first, middle and last frequencies of $3 \mathrm{~dB}$ gain bandwidth considering perpendicular planes of $\varphi=0$ and $\varphi=90^{\circ}$ are illustrated in Fig. 14 . 


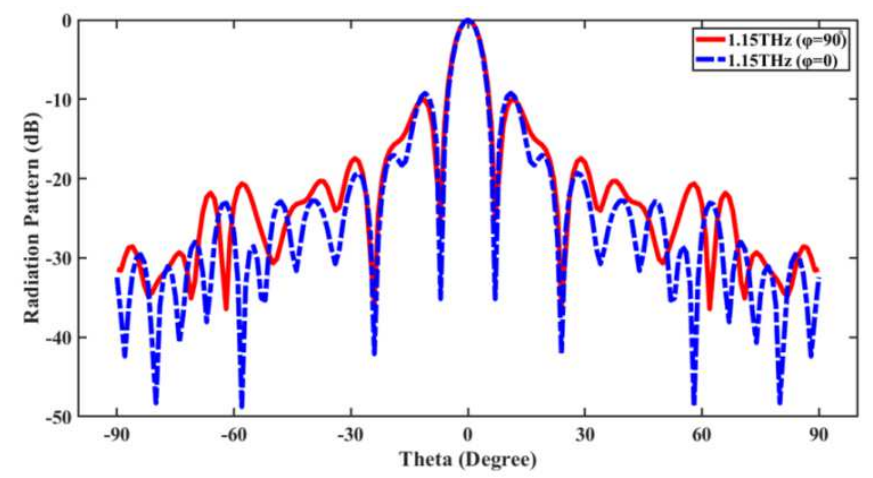

(a)

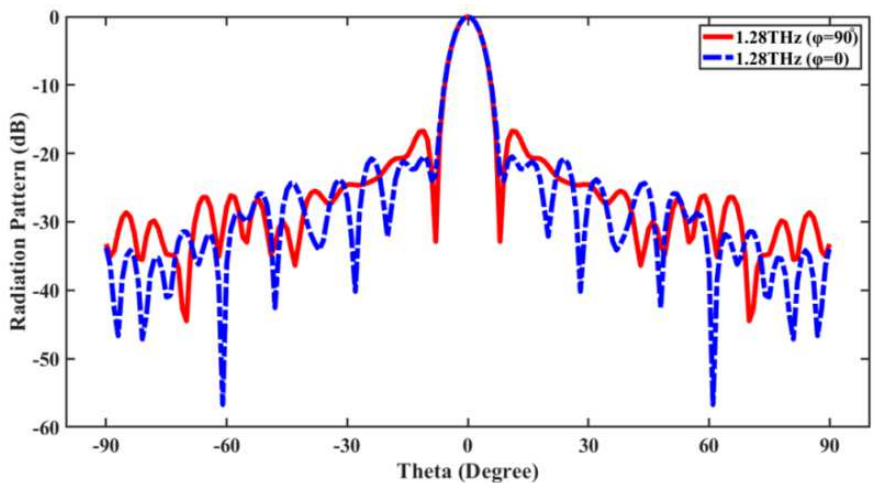

(b)

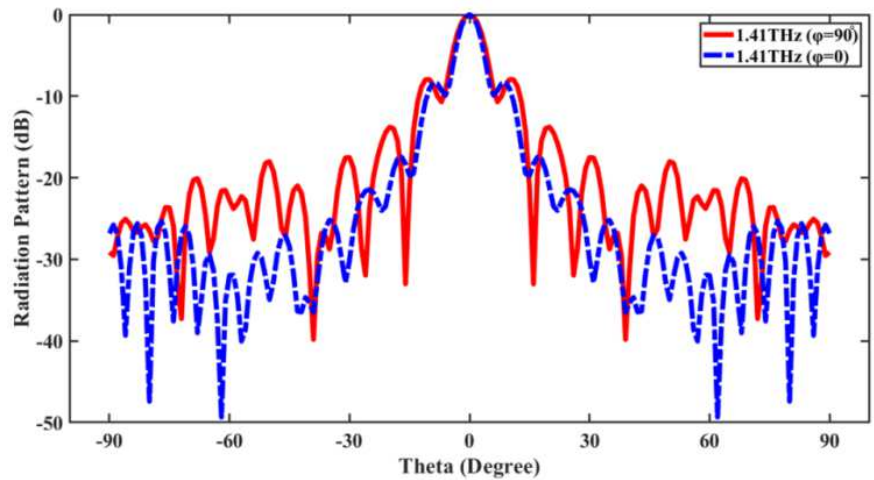

(c)

Fig. 14: Simulated radiation patterns of the designed square reflectarray antenna at (a) first, (b) middle and (c) last of frequencies of $3 \mathrm{~dB}$ gain bandwidth in Fig. 13

\section{5-3 Second reflectarray structure: circular array}

The schematic view of the reflectarray antenna with circular metasurface including of 277 optimized cells is depicted in Fig. 15. It should be noted that the design aspects of this antenna is the same as those of previous one, except that 84 lateral cells are removed to create a circular structure. 


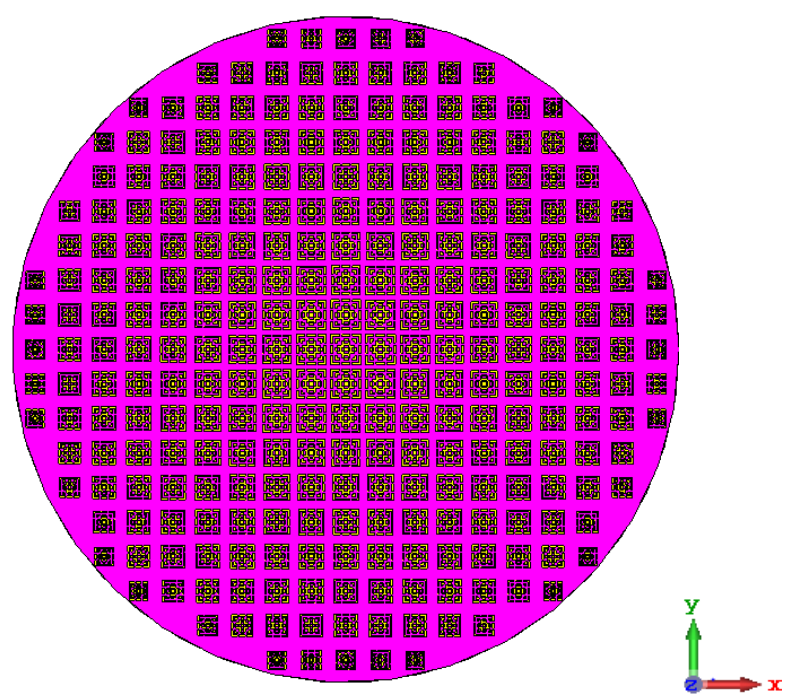

Fig. 15: Scheme of designed circular metasurface in the reflectarray antenna

In spite of reduced cell numbers in the circular reflectarray, the antenna gain is only $1 \mathrm{~dB}$ less than that of previous square reflectarray, and is equal to $24.9 \mathrm{~dB}$. However, the effective area of the circular aperture is $21 \%$ decreased resulting in an ultimately improved total efficiency. According to equation (8), the antenna efficiency at $1.3 \mathrm{THz}$ is $46.27 \%$, which is better than that of square structure. The comparison results of obtained gain with respect to horn feed antenna are presented in Fig. 16. The $3 \mathrm{~dB}$ gain bandwidth is $20.4 \%(1.19-1.46 \mathrm{THz})$.

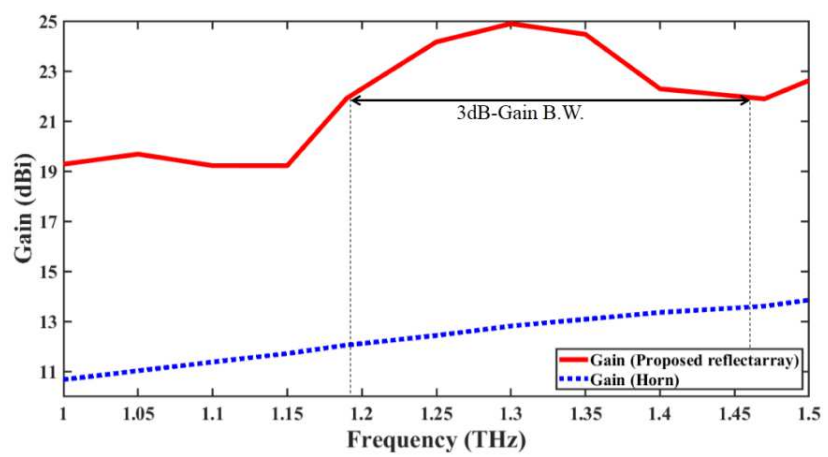

Fig. 16: Comparison results of gain variation of the designed circular reflectarray antenna and the horn feed antenna

The simulated radiation patterns of the circular reflectarray antenna at first, middle, and end frequencies of the corresponding $3 \mathrm{~dB}$ bandwidth considering two perpendicular planes of $\varphi=0$ and $\varphi=90^{\circ}$ are demonstrated in Fig. 17. 


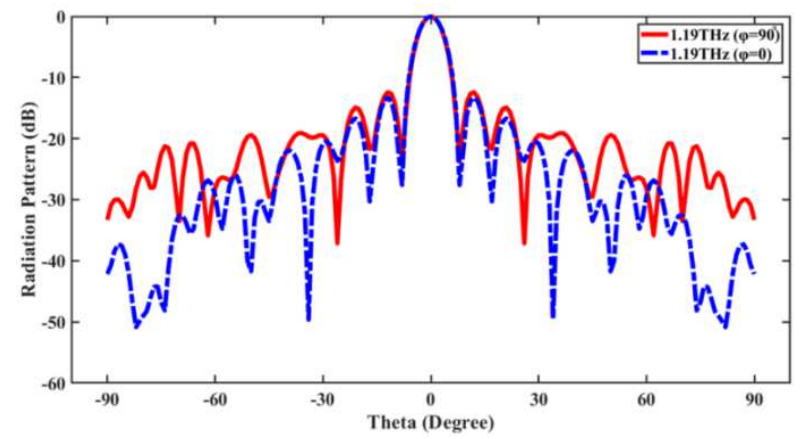

(a)

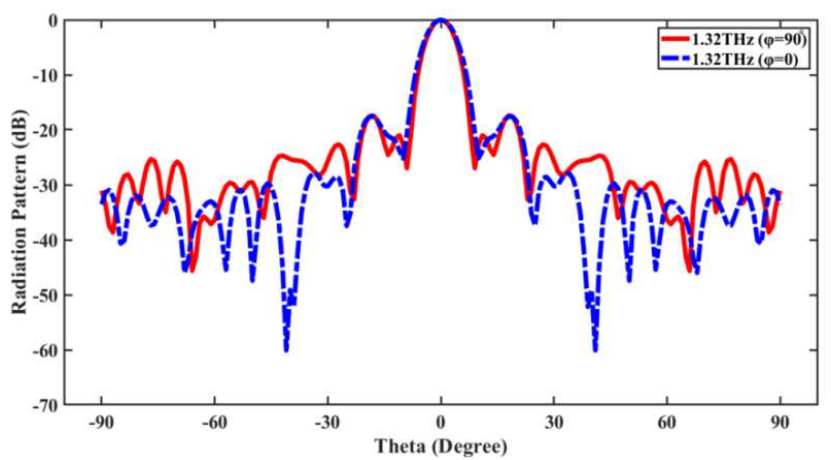

(b)

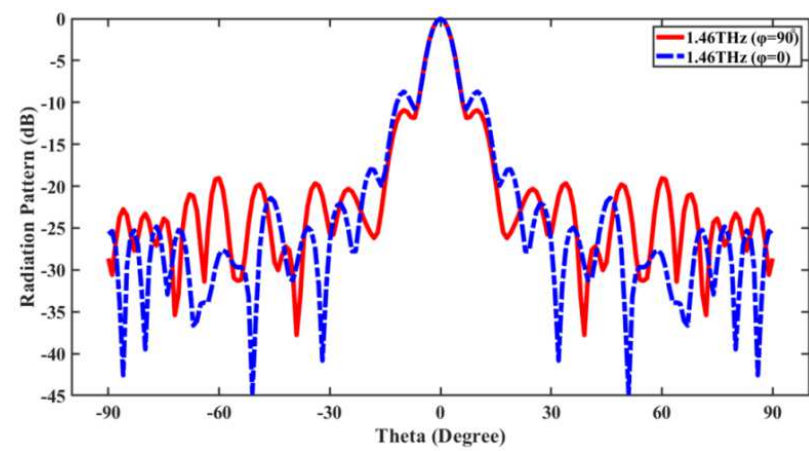

(c)

Fig. 17: Simulated radiation patterns of designed circular reflectarray antenna at (a) first, (b) middle, and (c) end frequencies of $3 \mathrm{~dB}$ gain bandwidth in Fig. 16

\section{5-4 Discussions}

First, the main characteristics of proposed cell are compared to some similar reported works in Table 4 . Then the two designed square and circular reflectarray antennas are compared to previous works in Table 5.

Table 4: Comparison between the properties of proposed unit cell and similar cells

\begin{tabular}{|c|c|c|c|c|}
\hline Reference & Frequency (THz) & Max $\left(\left|S_{11}\right|\right) \mathbf{d B}$ & Phase change range $\left(^{\circ}\right)$ & Optimization algorithm \\
\hline This work & $1-1.5$ & -0.42 & $\leq 738$ & $\checkmark$ \\
\hline$[31]$ & $0.9-1.17$ & - & $\leq 390$ & $\times$ \\
\hline$[10]$ & $0.357-0.421$ & -1.8 & $<550$ & $\times$ \\
\hline$[32]$ & $0.225-0.325$ & - & $\leq 550$ & $\times$ \\
\hline
\end{tabular}


Table 5: Comparison between the characteristics of designed reflectarray antennas and similar works

\begin{tabular}{|c|c|c|c|c|c|}
\hline Reference & $\begin{array}{c}\text { Feed } \\
\text { Antenna }\end{array}$ & Numbers of cells & Gain (dBi) & $\begin{array}{c}\text { Aperture Size } \\
\left(\mathrm{mm}^{2}\right)\end{array}$ & Efficiency(\%) \\
\hline $\begin{array}{c}\text { This work } \\
\text { (square layout) }\end{array}$ & horn & 361 & 25.9 & 3.61 & 45.67 \\
\hline $\begin{array}{c}\text { This work } \\
\text { (circular array) }\end{array}$ & horn & 277 & 24.9 & 2.83 & 46.27 \\
\hline$[31]$ & horn & - & - & 2500 & - \\
\hline$[10]$ & horn & - & 32.84 & 309.15 & 29.29 \\
\hline$[32]$ & horn & - & 33.98 & 400 & 43 \\
\hline
\end{tabular}

According to Table 4, the phase variation range of designed cell is highly improved in comparison to previous works. Moreover, the reflection coefficient value is less than reported one in [10]. As it is stated in Table 5, the efficiency of the designed square and circular antennas are considerably more than corresponding values in [10, $31,32]$. Since the aperture sizes of proposed arrays are much smaller in comparison to mentioned works, the resulting gain values are reduced.

\section{5-conclution}

In this manuscript, a single layer broadband unit cell with wide phase variation range was proposed. The RHC optimization algorithm and an established link between Matlab and HFSS are used to define and simulate various structures. A fitness function is defined in several stages to provide demanded goals including broadband and wide phase variation properties. Finally, a structure with maximum value of the objective function is chosen as an optimized one.

The optimized cell has wide phase variation range $\left(>600^{\circ}\right)$ in a broad frequency region between $1.1 \mathrm{THz}$ to 1.5 $\mathrm{THz}$ whereas the average magnitude of reflection coefficient is $-0.42 \mathrm{~dB}$. Two square and circular reflectarray antennas are implemented based on the optimized cell. According to simulation results, the square reflectarray antenna has 3-dB gain bandwidth of $20.3 \%$ and $45.67 \%$ efficiency, which are notable with respect to similar reported works. In the circular reflectarray antenna, the $3 \mathrm{~dB}$ gain bandwidth of $20.4 \%$ together with $46.27 \%$ efficiency are achieved. The maximum gain values of two square and circular antennas are $25.9 \mathrm{~dB}$ and $24.9 \mathrm{~dB}$, respectively.

Funding No funding was received to assist with the preparation of this manuscript.

Conflicts of interest The authors declare that they have no conflict of interest.

Availability of data and material The data and materials used to support the findings of this study are included within the article.

Code availability The written codes are available from the corresponding author on reasonable request.

Authors' contributions All authors have participated in design, simulation and preparation of the final version of the manuscript.

\section{References}

[1] Ferguson B, Zhang XC, (2002) Materials for terahertz science and technology. Nat. Mater. 1, 1: 26-33. 
[2] Nallappan K, Guerboukha H, Nerguizian C, Skorobogatiy M, (2018) Uncompressed HD and Ultra-HD video streaming using terahertz wireless communications. 11th Global Symposium on Millimeter Waves (GSMM), IEEE, 1-3.

[3] Deng R, Yang F, Xu S, Pirinoli P, (2014) Terahertz reflectarray antennas: An overview of the state-of-the-art technology. International Conference on Electromagnetics in Advanced Applications (ICEAA), IEEE, 667-670.

[4] Chaiyasoonthorn S, Limpaibool P, Mitatha S, Yupapin PP, (2010) High capacity mobile ad hoc network using THz frequency enhancement. International Journal of Communications, Network and System Sciences 3, 12: 954961.

[5] Khan MAK, Ullah MI, Kabir R, Alim MA, (2020) High-performance graphene patch antenna with superstrate cover for terahertz band application. Plasmonics, 15: 1719-1727.

[6] Song HJ, Nagatsuma T, (2011) Present and future of terahertz communications. IEEE Trans. Terahertz Sci. Technol. 1, 1: 256-263.

[7] Reid CB, Pickwell-MacPherson E, Laufer JG, Gibson AP, Hebden JC, Wallace VP, (2010) Accuracy and resolution of THz reflection spectroscopy for medical imaging. Physics in Medicine \& Biology 55, 16: 48254838.

[8] Yu C., Fan S., Sun Y., Pickwell-MacPherson E., (2012) The potential of terahertz imaging for cancer diagnosis: A review of investigations to date. Quantitative imaging in medicine and surgery 2, 1: 33-45.

[9] Huang J, Encinar J, (2008) Reflectarray Antenna. Wiley Online Library.

[10] Miao ZW, Hao ZC, Wang Y, Jin BB, Wu JB, Hong W, (2018) A 400-GHz high-gain quartz-based single layered folded reflectarray antenna for terahertz applications. IEEE Trans. Terahertz Sci. Technol. 9, 1: 78-88.

[11] Nayeri P, Liang M, Sabory-García R, Tuo M, Yang F, Gehm M, Xin H, Elsherbeni A, (2013) High gain dielectric reflectarray antennas for $\mathrm{THz}$ applications. IEEE Antennas and Propagation Society International Symposium (APSURSI), IEEE, 1124-1125.

[12] Peng B, Priebe S, Kürner T, (2014) Effects of phase shift errors on the antenna directivity of phased arrays in indoor terahertz communications. 11th International Symposium on Wireless Communications Systems (ISWCS), IEEE, 355-359.

[13] Wang H, Dong X, Yi M, Xue F, Liu Y, Liu G, (2017) Terahertz high-gain offset reflector antennas using SiC and CFRP material. IEEE Trans. Antenn. Propag. 65, 9: 4443-4451.

[14] Saha SC, Li C, Ma Y, Grant JP, Cumming DR, (2013) Fabrication of multilevel silicon diffractive lens at terahertz frequency. IEEE Trans. Terahertz Sci. Technol. 3, 4: 479-485.

[15] Gaufillet F, Marcellin S, Akmansoy E, (2016) Dielectric metamaterial-based gradient index lens in the terahertz frequency range. IEEE Journal of selected topics in quantum electronics 23, 4: 1-5.

[16] Yu Q, Gu J, Yang Q, Zhang Y, Li Y, Tian Z, Ouyang C, Han J, O'Hara JF, Zhang W, (2017) All-dielectric meta-lens designed for photoconductive terahertz antennas. IEEE Photonics Journal 9, 4: 1-9.

[17] Konstantinidis K, Feresidis AP, Constantinou CC, Hoare E, Gashinova M, Lancaster MJ, Gardner P, (2017) Low-THz dielectric lens antenna with integrated waveguide feed. IEEE Trans. Terahertz Sci. Technol. 7, 5: 572581.

[18] Lu G, Zhao R, Yin H, Xiao Z, Zhang J, (2020) Study of the Super Directive THz Photoconductivity Antenna. Plasmonics, https://doi.org/10.1007/s11468-020-01335-z.

[19] Pozar DM, Targonski SD, Syrigos H, (1997) Design of millimeter wave microstrip reflectarrays. IEEE Trans. Antenn. Propag. 45, 2: 287-296.

[20] Chang K, Wiley J, (2005) Encyclopedia of RF and microwave engineering. Wiley-Interscience.

[21] Hum SV, Okoniewski M, Davies RJ, (2005) Realizing an electronically tunable reflectarray using varactor diode-tuned elements. IEEE Microwave and Wireless Components Letters 15, 6: 422-424.

[22] Johnson RC, Jasik H, (1984) Antenna engineering handbook. McGraw-Hill.

[23] Berry D, Malech R, Kennedy W, (1963) The reflectarray antenna. IEEE Trans. Antenn. Propag. 11, 6: 645651. 
[24] Malagisi C, (1978) Microstrip disc element reflect array. Proc. Electronics and Aerospace Systems Convention, 186-192.

[25] Niu T, Withayachumnankul W, Ung BSY, Menekse H, Bhaskaran M, Sriram S, Fumeaux C, (2013) Experimental demonstration of reflectarray antennas at terahertz frequencies. Opt. Express 21, 3: 2875-2889.

[26] Niu T, Withayachumnankul W, Upadhyay A, Gutruf P, Abbott D, Bhaskaran M, Sriram S, Fumeaux C, (2014) Terahertz reflectarray as a polarizing beam splitter. Opt. Express 22, 13: 16148-16160.

[27] Sharifi R, Basiri R, Zareian-Jahromi E, (2020) Optimization-based design of a single-layer wideband reflectarray antenna in the terahertz regime. Journal of Computational Electronics 19, 1: 469-481.

[28] Skalak DB, (1994) Prototype and feature selection by sampling and random mutation hill climbing algorithms. Machine Learning Proceedings, 293-301.

[29] Deng R, Xu S, Yang F, Li M, (2016) A single-layer high-efficiency wideband reflectarray using hybrid design approach, IEEE Antennas Wirel. Propag. Lett. 16, 884-887.

[30] Nayeri P, Yang F, and Elsherbeni AZ, (2018) Reflectarray Antennas: Theory, Designs and Applications Wiley Online Library.

[31] You X, Ako RT, Lee WS, Low MX, Bhaskaran M, Sriram S, Fumeaux C, Withayachumnankul W, (2019) Terahertz reflectarray with enhanced bandwidth. Advanced Optical Materials 7, 20: 1900791:1-8.

[32] Qu SW, Wu WW, Ng KB, Chen BJ, Chan CH, Pun EYB, (2014) Wideband terahertz reflectarrays with fixed/frequency-scanning beams. XXXIth URSI General Assembly and Scientific Symposium (URSI GASS), IEEE, $1-4$. 